\title{
分子印迹聚合物固相萃取研究进展
}

\author{
李金花 ${ }^{1}$ ，温莹莹 ${ }^{1,2}$ ，陈令新 ${ }^{1 *}$
}

(1. 中国科学院烟台海岸带研究所, 山东 烟台 264003；2. 中国科学院大学, 北京 100049)

\begin{abstract}
摘要: 对最新报道的分子印迹聚合物作为固相萃取剂及其在色谱样品前处理方面的应用进行综述和展望, 主要包 括固相萃取、基质固相分散萃取、固相微萃取、搅拌棒吸附萃取和磁性材料萃取, 同时总结了分子印迹聚合物制备 技术面临的挑战和问题,提出了可能的解决方案。
\end{abstract}

关键词: 分子印迹聚合物; 固相萃取; 固相吸附材料; 色谱

中图分类号: O658 文献标识码:A 文章编号:1000-8713(2013)03-0181-04

\section{Advances of molecularly imprinted polymers for solid phase extraction}

\author{
LI Jinhua ${ }^{1}$, WEN Yingying ${ }^{1,2}$, CHEN Lingxin ${ }^{1 *}$ \\ (1. Yantai Institute of Coastal Zone Research, Chinese Academy of Sciences, Yantai 264003, China; \\ 2. University of Chinese Academy of Sciences, Beijing 100049, China)
}

\begin{abstract}
Recent advances in molecularly imprinted polymers (MIPs) as solid phase adsorbents for chromatographic sample pretreatments are summarized, including solid phase extraction, matrix solid phase dispersion extraction, solid phase microextraction, stir bar sorptive extraction and magnetic material extraction. A number of challenges and possibly effective strategies are discussed. And some perspectives of MIPs based extraction in chromatography are also proposed.
\end{abstract}

Key words : molecularly imprinted polymer; solid phase extraction; solid phase adsorbent; chromatography

分子印迹技术是指制备对某一特定目标分子具 有特异选择性的聚合物即分子印迹聚合物 ( molecularly imprinted polymers, MIPs) 的程序, 常被形 象地描绘为制造识别 “分子钥匙” 的 “人工锁” 的技 术。分子印迹最先由 Polyakov 在 1931 年提出, 20 世纪 80 年代后发展迅速 ${ }^{[1]}$, 现阶段在萃取/分离、化 学/生物传感和人工抗体等方面应用广泛。本研究 组 ${ }^{[2,3]}$ 综述了分子印迹在上述各方面的研究及应 用, 李攻科研究组 ${ }^{[4]}$ 综述了近年来分子印迹微萃取 技术的研究进展, 唐和清研究组 ${ }^{[5]}$ 综述了基于分子 印迹的高毒性有机污染物的去除。MIPs 材料作为 固相吸附剂在色谱中的应用主要体现在环境、食品、 生物等样品的前处理中 (固相萃取、基质固相分散 萃取、固相微萃取、搅拌棒吸附萃取和磁萃取等多方 面)。本文就此综述了近 3 年来 MIPs 作为固相萃
取剂的研究新进展, 并总结了目前分子印迹制备技 术的挑战和解决方案, 最后对其作为固相萃取材料 进行了展望。

\section{MIPs 在萃取中的应用}

固相萃取 (solid phase extraction, SPE) : 这是 利用固体吸附剂对液体试样中各组分的吸附力差异 而实现待测组分和干扰组分分离的技术。固相吸附 剂对目标分析物的选择性越高, 则分离效率越高。 因此, 采用高选择性的 MIPs 作为 SPE 吸附剂, 可实 现目标分析物的高效萃取富集 ${ }^{[2,6]}$ 。Xu 等 ${ }^{[7]}$ 将多 孔聚合物与分子印迹相结合, 制备了多孔中空、单孔 中空和多孔实心 3 种莠去津多孔结构的 $M I P s 。$ 并 将单孔中空结构莠去津印迹聚合物作为 SPE 填料 用于土壤加标样品中 5 种三嗪类除草剂的高选择性 
浓缩富集, 通过高效液相色谱 (HPLC) 检测, 加标回 收率为 $94.5 \% \sim 106 \%$, 相对标准偏差 (RSD) 为 $1.17 \% \sim 4.24 \%$ 。该类多孔 MIPs 的制备和应用, 为 复杂样品基质中三嗪的分离富集提供了一种有效方 法。Zhang 等 ${ }^{[8]}$ 通过多步溶胀聚合合成单孔中空 MIPs, 并将其用作 SPE 填料来选择性地萃取苏丹红 染料获得了满意的结果, 实现了辣椒酱中苏丹红的 测定。该方法检出限为 3.3 和 $5.0 \mu \mathrm{g} / \mathrm{kg}$, 回收率 为 $89.7 \% \sim 102.0 \%$ 。Song 等 ${ }^{[9]}$ 采用溶胶-凝胶表面 印迹方法通过非共价聚合, 以 16 种多环芳烃 (PAHs) 为模板分子, 成功合成了具有高选择性的 MIPs, 并以此作为 SPE 填料来萃取海水中的 $\mathrm{PAHs}$, 用气相色谱-质谱 (GC-MS) 作为检测手段。 结果表明,该法对于海水中 PAHs 的检测是一种选 择性好、准确度高、灵敏度高的方法。Peng 等 ${ }^{[10]}$ 将 甲基甲磺隆印迹聚合物包裏在硅纳米球上制备纳米 级的固相吸附材料。采用分散固相萃取选择性地萃 取了土壤、大米、大豆和玉米中的硫脲类除草剂。此 法简单、快速、回收率高。

基质固相分散萃取 (matrix solid phase dispersion, MSPD) : 这是一种新型的样品前处理技术,集 样品提取、净化于一体，既避免了样品损失又节省了 溶剂,具有操作简便快捷、提取效率高、无需特殊设 备等优点,并可进行大批量自动化分析,因而广泛应 用于药物残留、污染物和有害成分的分析。MIPs 作 为一种选择性高的固相吸附剂应用于 MSPD 中为 复杂基质中污染物及有害成分的分析提供了有力的 技术支撑。Wen 等 ${ }^{[11]}$ 利用经典聚合方法一一本体 聚合合成了以莠去津为模板分子的 MIPs, 并将其用 作 MSPD 的分散剂萃取土壤、西红柿和草莓中的莠 去津、扑灭津等三嗪类除草剂, 用胶束电动色谱 (MEKC) 检测。在最佳的萃取和检测条件下, 实现 了三嗪类除草剂高选择性、高灵敏度的检测。方法 检出限在 $12.9 \sim 31.5 \mathrm{ng} / \mathrm{g}$ 之间, 回收率为 $65.1 \%$ ～97. 4\%。此法简单、快速, 并适用于多种复杂基质 如土壤、蔬菜和水果中除草剂的选择性萃取和检测。

固相微萃取 (solid phase microextraction, SPME) : 这是以 SPE 为基础研发的一种无溶剂前处 理方法,易与 GC、HPLC 和 CE 联用,从而得到广泛 的应用,已成功应用于气体、水体、土壤、底泥等环境 样品中有机物甚至无机物的分析中。SPME 过程实 际上是样品中各组分在萃取纤维涂层表面吸附/解 吸附过程,通过选择固载不同基团的涂层的萃取头, 使目标组分得到最佳萃取效率而其他组分受到抑 制。其中 MIPs 涂层由于制备简单、能够反复使用、
机械强度较高、耐高温和耐溶剂性好, 尤其是具有高 效选择特异性的优点, 可适用于复杂环境介质中的 痕量目标物分析, 应用前景广阔, 已成为选择性 SPME 涂层的研究热点之一。Golsefidi 等 ${ }^{[12]}$ 通过 溶胶-凝胶方法合成了氯原酸 MIPs, 在印迹合成过 程中还加人了多壁碳纳米管来改善印迹材料的吸附 速率。将 MIPs 聚合在中空纤维膜 ( HF) 上用于 SPME 装置, 高效、快速地萃取药物样品中的氯原 酸。该 MIP-HF-SPME 法获得了较低检出限 $(0.08$ $\mathrm{ng} / \mathrm{mL}$ ), 实现了氯原酸的高选择性、高灵敏度检测。

搅拌棒吸附萃取 (stir bar sorptive extraction, SBSE) : 这是一种新型的环境友好型样品前处理技 术,先使目标化合物在样品基质和搅拌棒表面涂敷 的高分子材料涂层之间达到分配平衡, 然后利用热 脱附或溶剂脱附技术进样分析。目前广泛采用的涂 层材料为聚二甲基硅烷 (PDMS), 这种采用溶胶-凝 胶法制备的涂层结构紧密,且高度疏水,化学性质稳 定,适用于水相中非极性和弱极性化合物的萃取。 黄晓佳研究组 ${ }^{[13]}$ 综述了搅拌棒固相萃取的研究进 展。但是由于 PDMS 涂层种类单一, 而且选择性不 高,所以各种新型的 SBSE 涂层应运而生。具有高 选择性的 MIPs 材料就成为 SBSE 的重要涂层之一。 他们 ${ }^{[14]}$ 以双酚 $\mathrm{A}(\mathrm{BPA})$ 为模板分子, 利用整体材料 “原位”聚合技术制备了 BPA-MIPs 萃取搅拌棒, 然 后与 HPLC 联用,对环境水样中的 BPA 进行选择性 地萃取。在最佳的萃取条件下, MIP-SBSE 可对 BPA 进行有效的选择性吸附, 线性范围为 $1.0 \sim 200$ $\mu \mathrm{g} / \mathrm{L}$, 检出限为 $0.28 \mu \mathrm{g} / \mathrm{L}$, 回收率为 $96.0 \% \sim$ $108.7 \%$ 。 $\mathrm{Hu}$ 等 ${ }^{[15]}$ 通过化学键合把三嗪类除草剂 MIPs 键合到玻璃摚拌棒上, 并用此摚拌棒成功萃取 了大米、苹果、卷心菜和土壤中的三嗪类除草剂, 方 法检出限在 $0.04 \sim 0.12 \mu \mathrm{g} / \mathrm{L}$ 之间, 回收率为 72. $0 \% \sim 114.8 \%$ 。

磁性材料萃取 (magnetic material extraction, $\mathrm{MME}$ ) : 将 MIPs 修饰在磁性纳米微球 (如: $\mathrm{Fe}_{3} \mathrm{O}_{4}$ 磁 性微球) 表面就形成了分子印迹磁性微球。这种材 料在使用时通过磁性分离就可以将吸附了目标化合 物的微球和样品基质快速分离, 使整个样品前处理 过程变得简单易行。李攻科研究组合成了多种分子 印迹磁性微球作为样品前处理的吸附材料 ${ }^{[16-18]}$, 并 详细总结了分子印迹磁性微球在样品前处理方面的 应用 ${ }^{[4]}$ 。王志研究组 ${ }^{[19]}$ 也对其进行了综述。Xu 等 ${ }^{[20]}$ 通过结合分子印迹技术与刺激响应聚合物, 制 备了一种新型的光-磁双重响应的分子印迹聚合物 (DR-MIPs), 该聚合物具有传质快、吸附容量高、选 
择性好、易分离等优势。简单、快速、低成本且环境 友好的 MIPs 磁萃取方法在复杂基质痕量分析中具 有巨大的应用潜力。

目前, MIPs 作为固相吸附剂主要用于萃取环 境、食品和生物等样品中的目标分析物。其中环境 水、土壤等介质中的莠去津 ${ }^{[7,21]}$ 、雌激素类化合 物 ${ }^{[22]}$ 和双酚 $\mathrm{A}^{[23]}$ 等在环境基质中研究较多; 食品 样品种类繁多, MIPs 作为固相吸附剂用于食品中目 标分析物的检测近年来备受关注,例如: 卷心菜、向 日葵籽和茶叶中的氯乙酰胺类农药 ${ }^{[24]}$ 、麦片中毒枝 震素 ${ }^{[25]}$ 和牛奶中氧四环素 ${ }^{[26]}$ 的选择性萃取及测定 等。生物样品中关于血清和尿液的研究较多, 例如 溴芐环己铵的 MISPE 分析 ${ }^{[27]}$, 麻黄素和麻黄碱的 MI-SPME 分析 ${ }^{[28]}$, 氟喹诺酮类和黄嘌呤类衍生物 的 MI-MSPD 分析 ${ }^{[29]}$ 等。

\section{MIPs 制备技术的挑战及解决方案}

尽管目前各种新型的 MIPs 不断出现并且得到 广泛应用,但仍然在制备技术上面临诸多挑战和问 题,解决它们势在必行。

印迹容量低和功能单体单一:分子印迹技术发 展中遇到的最大问题仍然是印迹容量低, 主要原因 在于 MIPs 的制备过程中使用大量交联剂(通常的 物质的量比为模板：单体：交联剂 $=1: 4: 20)$ 。大量 交联剂的使用使得单位质量印迹聚合物中的识别位 点数量有限, 且位点的利用率较低。此外, 传统本体 聚合方法得到的 MIPs 往往具有颗粒不均匀、形态 不规则等缺点从而导致传质慢, 结合量低。解决这 一问题的途径通常有两条: (1) 结合纳米技术, 制备 纳米级且形状规则、粒径均一的印迹微球 ${ }^{[30,31]}$; (2) 采用表面印迹技术, 制备超薄壳层的印迹材 料 ${ }^{[9,30-32]}$ 。CaO 等 ${ }^{[31]}$ 将聚苯胺固定在印迹纳米纤 维表面,通过洗脱除去模板分子,在纳米纤维聚合物 表面上留下识别孔穴。研究结果表明,该表面印迹 聚合物的印迹容量大大提高, 并可以应用在生物分 子或者生物传感器领域。

功能单体单一也限制了 MIPs 的发展。通用的 功能单体仍局限于甲基丙烯酸、乙烯基吡啶、丙烯酰 胺这几种 ${ }^{[2]}$ 。这些单体对很多模板分子缺少特异 性的高亲和力的结合位点, 从而造成所制备的 MIPs 结合容量较低。根据具体的模板分子设计合成专一 性功能单体可以解决上述问题 ${ }^{[33]}$

水相识别: 目前多数 MIPs 的制备和应用都局 限在有机溶剂中进行, 而天然的识别体系以及 MIPs 所面临的实际应用环境则多是水性体系。水环境下
的分子识别问题仍是一个较大的挑战,存在于两个 方面:MIPs 在水相中不均匀分散的问题和 MIPs 可 以识别水相中目标物的问题。解决其在水相中均匀 分散的问题可以采用的方案是对其表面进行亲水性 改性,如接枝亲水性的聚合物刷。解决不可以水相 识别的问题一般要采用金属鳌合作用力、疏水作用 力等不受水干扰的作用力进行印迹, 这就对功能单 体提出了要求; 另外,识别微环境最好与聚合微环境 相一致, 聚合条件尽量温和,不破坏聚合位点,紫外 聚合可重现常温的预结合与再识别,防止高温破坏。 与有机介质不同的是, 由于水分子的参与,水环境下 的分子识别问题要复杂得多,水溶液的组成、 $\mathrm{pH}$ 值、 溶液中盐的离子强度、极性有机溶剂加人的比例和 种类等,均会对作用双方产生显著的影响。Watabe 等 ${ }^{[34]}$ 以对叔丁基苯酚为替代模板,制备了尺寸均一 的分子印迹固相萃取剂进行在线预处理, 检出限达 $0.36 \mathrm{ng} / \mathrm{L}$, 成功应用于环境水样中双酚 $\mathrm{A}$ 的测定。

大分子识别: 现阶段 MIPs 的研究重点从亲脂 性的有机小分子逐渐转移到有机大分子, 特别是多 肽、蛋白质等生物大分子, 甚至整个细胞的印迹。但 是蛋白质分子结构复杂, 与功能单体的结合位点多, 这就造成了功能单体选择困难; 另外,巨大的分子体 积使其在印迹聚合物中传质较差, 不易洗脱; 而且蛋 白质在许多条件下易发生变性失活,空间结构改变。 因此,蛋白质聚合物合成条件较为苛刻, 功能单体、 交联剂、溶剂、聚合温度等条件的选择对合成高选择 性 MIPs 十分重要。 $\mathrm{He}$ 等 $^{[35]}$ 及 Jing 等 ${ }^{[36]}$ 在二氧 化硅球表面成功制备了溶菌酶分子印迹聚合物,所 制备的 MIPs 对模板蛋白分子有良好的选择性。

\section{3 结论与展望}

综上所述, 具有构效预定性、特异识别性和广泛 适用性的新型 MIPs 的不断制备和发展必将为复杂 样品的前处理手段提供很好的技术平台,而且快速、 灵敏的色谱检测手段的发展也为分析物的检测提供 了有利的工具, 这将更加促进 MIPs 作为固相吸附 剂在各领域样品前处理中的应用, 为实现目标物的 高选择性、高灵敏度检测奠定坚实基础。此外, MIPs 也正在被越来越多地用作液相色谱柱的填料 (比如某些分子印迹预处理柱和整体柱的制备等) 用于在线分析。但是, MIPs 作为 MSPD 和 SBSE 的 固相吸附剂的研究还不是非常全面,这就对分子印 迹技术在样品前处理的应用方面提出了新的挑战; 其次, 为了实现微型化、高效、快速、准确和高选择性 地萃取复杂基质中的目标分析物, 对 SPE 和 SPME 
进行适当的改良是 MIPs 在样品前处理应用方面面 临的另一挑战。分析工作者应积极应对挑战并找到 有效的解决策略,深人拓展分子印迹固相吸附剂在 色谱样品前处理方面的应用。

\section{参考文献:}

[1] Verheyen E, Schillemans J P, van Wijk M, et al. Biomaterials, 2011, 32: 3008

[2] Chen L X, Xu S F, Li J H. Chem Soc Rev, 2011, 40: 2922

[3] Wen Y Y, Li J H, Ma J P, et al. Electrophoresis, 2012, 33 : 2933

[4] Zhang K G, Hu Y L, Hu Y F, et al. Chinese Journal of Chromatography (张凯歌, 胡玉玲, 胡玉斐, 等. 色谱), 2012, 30 (12) : 1220

[5] Shen X T, Zhu L H, Wang N, et al. Chem Commun, 2012, 48: 788

[6] Song X L, Li J H, Wang J T, et al. Talanta, 2009, 80: 694

[7] Xu S F, Chen L X, Li J H, et al. J Mater Chem, 2011, 21: 12047

[8] Zhang Z, Xu S F, Li J H, et al. J Agric Food Chem, 2012, $60: 180$

[9] Song X L, Li J H, Xu S F, et al. Talanta, 2012, 99: 75

[10 ] Peng Y, Xie Y, Luo J, et al. Anal Chim Acta, 2010, 674: 190

[11] Wen Y Y, Chen L X, Li J H, et al. Electrophoresis, 2012, $33: 2454$

[12] Golsefidi M A, Es' haghi Z, Sarafraz-Yazdi A. J Chromatogr A, 2012, 1229: 24

[13 ] Chen L L, Huang X J, Yuan D X. Chinese Journal of Chromatography (陈林利, 黄晓佳, 袁东星. 色谱), 2011, 29 (5) : 375

[14] Lin F H, Huang X J, Yuan D X. , et al. Chinese Journal of Chromatography (林福华, 黄晓佳, 袁东星, 等. 色谱), $2010,28(5): 507$

[15] Hu Y L, Li J W, Hu Y F, et al. Talanta, 2010, 82: 464

[16] Zhang Z M, Tan W, Hu Y L, et al. J Chromatogr A, 2011,

\section{8: 4275}

[17] Zhang Y, Liu R J, Hu Y L, et al. Anal Chem, 2009, 81: 967

[18 ] Hu Y L, Li Y W, Liu R J, et al. Talanta, 2011, 84: 462

[19] Wang Z, Wang C. Chinese Journal of Chromatography (王 志, 王春. 色谱), 2012, 30(10) : 977

[20 ] Xu S F, Li J H, Song X L, et al. Anal Methods, 2013, 5: 124

[21] Djozan D, Ebrahimi B, Mahkam M, et al. Anal Chim Acta, 2010, 674: 40

[22] Prieto A, Vallejo A, Zuloaga O, et al. Anal Chim Acta, 2011, 703: 41

[23] Yin Y M, Chen Y P, Wang X F, et al. J Chromatogr A, $2012,1220: 7$

[24] Zhang L, Han F, Hu Y Y, et al. Anal Chim Acta, 2012, $729: 36$

[25] Ali W H, Derrien D, Alix F, et al. J Chromatogr A, 2010, 1217: 6668

[26] Lv Y K, Wang L M, Yang L, et al. J Chromatogr A, 2012, 1227: 48

[27] Javanbakht M, Namjumanesh M H, Akbari-Adergani B. Talanta, 2009, 80: 133

[28 ] Deng D L, Zhang J Y, Chen C, et al. J Chromatogr A, 2012, 1219: 195

[29] Qiao F X, Yan H Y. J Chromatogr B, 2011, 879 : 3551

[30 ] Xu S F, Li J H, Chen L X. J Mater Chem, 2011, 21: 4346

[31] Cao F M, Liao J, Yang K G. Anal Lett, 2010, 43 : 2790

[32] Li J H, Zhang Z, Xu S F, et al. J Mater Chem, 2011, 21: 19267

[33 ] Xu S F, Chen L X, Li J H, et al. J Hazard Mater, 2012, 237/238: 347

[34 Watabe Y, Kondo T, Morita M, et al. J Chromatogr A, 2004, 1032: 45

[35] He H Y, Fu G Q, Wang Y, et al. Biosens Bioelectron, $2010,26: 760$

[36] Jing T, Du H R, Dai Q, et al. Biosens Bioelectron, 2010, 26: 301 